La traducción médica es una de las actividades más presentes en el entorno profesional, pero también una de las menos investigadas. La singularidad del campo hace que sea deseable la creación de recursos documentales específicos para traductores y redactores médicos. Por ello, es recomendable analizar las expectativas que tiene esta comunidad profesional respecto al uso de fuentes documentales. En este artículo se presentan y discuten los resultados de una encuesta elaborada por el grupo de investigación Gentt y difundida entre traductores médicos en España, a partir de los cuales se proponen futuras líneas de trabajo.

PALABRAS CLAVE: traducción, ámbito médico, investigación cualitativa, comunidad socioprofesional, género

\title{
Necesidades documentales del traductor médico en España
}

ISABEL GARCIA-IZOUIERDO

Universitat Jaume I

Grupo GENTT

TOMÁs CONDE

Universidad del País Vasco

Grupo GenTT

\section{Documentation Needs of Medical Translators in Spain}

Medical translation is one of the most present - and least investigated - activities in the professional environment. The singularity of the field makes it necessary to create specific documentary resources appropriate for medical translators and writers. Thus, it is advisable to analyze the expectations that this professional community has with regard to the use of documentary sources. This article presents and discusses the results of a survey designed by the Gentt Research Group and administered to medical translators in Spain. Finally, several lines of research are proposed on the basis of such results.

KEY WORDS: translation, medical field, qualitative research, socio-professional community, genre 


\section{INTRODUCCIÓN}

En los últimos años se ha puesto de manifiesto la necesidad de ahondar en el estudio de la traducción médica como un campo específico de traducción (Lee-Jahnke, I998), uno de los tipos más productivos por lo que respecta a la práctica profesional y, no obstante, también uno de los menos investigados (Montalt and González, 2007). ${ }^{\mathrm{I}}$ En trabajos anteriores (García Izquierdo, 20II $)^{2}$ se ha defendido la utilidad de realizar investigaciones cualitativas, mediante encuestas, para conocer la opinión de los traductores expertos en determinados ámbitos de especialidad con el objetivo de aplicar los resultados obtenidos, en combinación con los ofrecidos por la investigación cuantitativa, a la creación de herramientas de trabajo que faciliten a los sujetos de las comunidades profesionales estudiadas la redacción/traducción de la documentación con la que trabajan. ${ }^{3}$

Ahora bien, en el ámbito médico se constata la necesidad de profundizar en la investigación acerca de los procesos documentales y el diseño de recursos para los traductores y redactores de manera específica, ${ }^{4}$ especialmente en el contexto español, donde la tradición de este tipo de traducción está, en muchas ocasiones, en manos de traductores no profesionales o de especialis-

r Esta situación está comenzando a revertirse con la aparición reciente de publicaciones monográficas centradas en el ámbito médico (véase, por ejemplo, Montalt y Shuttleworth [eds.], 20I2).

2 Investigación desarrollada en el seno del equipo Gentt (www.gentt.uji.es), con la financiación de la Universitat Jaume I y la Fundación Caixa-Castelló.

3 Los resultados globales de esta investigación, con el análisis comparativo de los datos de los diferentes ámbitos y las repercusiones desde la perspectiva de la traducción se pueden consultar en García Izquierdo y Conde, 2012.

4 Esta es la opinión, por ejemplo, de Borja (2012: I67), cuando afirma que tanto las dificultades que presenta la traducción de determinados textos médico-jurídicos como las competencias exigibles a sus traductores pueden ser específicas. tas del ámbito médico y donde la comunidad socioprofesional (y su configuración, por tanto) está menos convencionalizada (Muñoz Miquel, Montalt y García Izquierdo, 20II) que otras como, por ejemplo, la de los traductores jurídicos.

En el presente artículo profundizaremos, pues, en los resultados para el ámbito médico del estudio cualitativo arriba citado a fin de analizar sus posibles repercusiones y proponer líneas de investigación futuras.

\section{MATERIALES Y MÉTODOS}

Los resultados del análisis de las necesidades en los diferentes ámbitos de especialidad (jurídico, médico y técnico) sacaron a la luz diferencias (García Izquierdo y Conde, 20I2), y pusieron de manifiesto la necesidad de orientar el diseño de recursos de manera específica.

Como se expone en García Izquierdo (20II, 603 ss.), la investigación se diseñó utilizando LimeSurvey, un programa de encuestas en línea y gratuito, de fácil manejo además, que permite el envío de un número ilimitado de encuestas, con un número ilimitado de preguntas y un número ilimitado de participantes y que incluye utilidades para el tratamiento estadístico de los datos. La encuesta para el ámbito médico constaba de tres grandes bloques:

I. E1 bloque A, denominado «Traducción e Interpretación médica (I)», incluía preguntas relacionadas con los ámbitos específicos dentro de la especialidad de los que se traducía y a los que se traducía; el tipo de cliente para el que se traducía; los géneros de los que se traducía y a los que se traducía (utilizando para este propósito el árbol de géneros diseñado por el equipo para cada uno de los tres ámbitos —véase www.gentt.uji.es); y las lenguas habituales de trabajo. 
2. E1 bloque $B$, denominado «Traducción e interpretación médica (II)», incluía preguntas relacionadas con los recursos de consulta documental utilizados habitualmente por los informantes y el formato de dichos recursos; información que se desearía obtener de los mismos; motivación para las consultas terminológicas, etc.

3. El bloque $\mathrm{C}$, denominado «Situación profesional», incluía preguntas relativas al género y el sexo del informante, su formación, su experiencia en años como traductor o el tipo de trabajo desempeñado.

Por lo que respecta a los informantes, ${ }^{5}$ para elaborar el censo de traductores médicos se recurrió a tres fuentes diferentes:

- la Asociación Internacional de Traductores y Redactores de Medicina y Ciencias afines, Tremédica (http://www.medtrad.org/); (30)

- el foro de Medicina y Traducción Medtrad, perteneciente a la asociación anterior (II), y

- el Máster en Traducción Médico-Sanitaria de la Ujı. (68)

De esta manera elaboramos el censo formado por rog traductores médicos de entre los que se seleccionó una muestra aleatoria (30 \% de la población) que dio como resultado un grupo de I8 informantes ( $65 \%$ de respuestas).

Somos conscientes del carácter no representativo de los resultados, pero consideramos que, aun así, puede resultar interesante detenernos en el análisis específico de este ámbito tan poco estudiado. Por ello, en las líneas que siguen, expondremos los resultados más significativos obtenidos a partir de la consulta a estos informantes.

\footnotetext{
5 Para ampliar esta información, véase García Izquierdo (20II).
}

\section{RESULTADOS Y DISCUSIÓN}

Se presentan las respuestas en dos grandes bloques: en primer lugar, los aspectos relacionados con el perfil de los sujetos (lo que nos puede 143 ayudar a corroborar la menor consistencia de la comunidad socioprofesional de la que hablábamos en líneas anteriores); y, en segundo lugar, las respuestas a cuestiones como el espectro de géneros con el que trabajan, las lenguas de trabajo o los recursos documentales que utilizan. Finalmente, se realiza una reflexión, utilizando los datos obtenidos, acerca de la relación entre la pericia ${ }^{6}$ de los traductores entrevistados y su desempeño profesional.

\section{Perfil de los sujetos}

\section{I.I. Edad y sexo}

$\mathrm{El}$ informante más joven cuenta con 28 años y el de más edad, con 69. La gran mayoría de los sujetos del estudio son treintañeros o han superado los cuarenta recientemente. En cuanto al sexo, prácticamente 2 de cada 3 informantes (II de I8) eran mujeres. Un sujeto prefirió no especificar su sexo. El gráfico I muestra la frecuencia de edades y géneros.

Se puede comprobar cómo los sujetos femeninos (que eran más numerosos) abarcan un abanico de edad más amplio que el de los sujetos masculinos. En un caso y otro, sin embargo, la mayoría de sujetos rondan la cuarentena. Habría que interpretar estos datos con cautela, habida cuenta de que muchos de nuestros informantes eran bien estudiantes del máster, bien profesionales jóvenes que buscaban reciclarse; en consecuencia, no creemos conveniente gene-

6 Seguimos la definición de pericia de Ericsson (I996, apud Muñoz, 20II), que consiste en el conjunto de capacidades características de los expertos, esto es, de las personas que ejecutan una acción en un ámbito concreto con resultados excepcionales. 


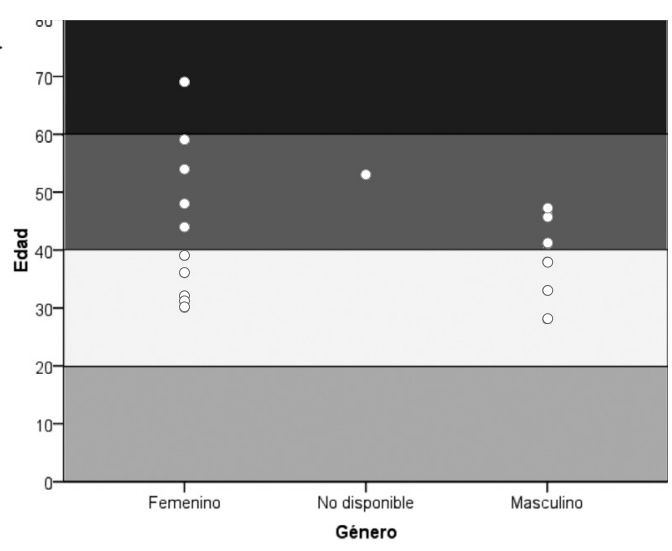

Gráfico I. Edad y género de los informantes ralizar estos resultados a toda la población de traductores médicos.

\section{I.2. Nivel de estudios}

En la tabla I figuran los estudios que los sujetos dicen tener en su haber. Se puede apreciar que muchos se han acercado al ámbito de la traducción médica probablemente porque en su formación han realizado estudios superiores tanto de traducción como de ciencias. Entre los que no responden a este perfil (Io), dos son filólogos y expertos en ciencias, con lo que más de la mitad de los informantes son expertos en lenguas (sobre todo en traducción) y alguna rama de la ciencia. Casi el $80 \%$ tienen estudios superiores en Traducción, y entre los cuatro que no los tienen, uno es filólogo.

TABLA I. ESTUDIOS DE LOS INFORMANTES

\begin{tabular}{|c|c|c|c|c|c|c|c|}
\hline Sujeto & Traducción & Filología & Medicina & Biología & Farmacia & Veterinaria & Otra \\
\hline$I$ & Licenciatura & & & & & & \\
\hline 2 & & & & Licenciatura & & & Máster \\
\hline 3 & & & Máster & & & & \\
\hline 4 & Licenciatura & & Máster & & Máster & & \\
\hline 5 & & & Licenciatura & & & & Licenciatura \\
\hline 7 & Diplomatura & & & & & & \\
\hline 9 & Licenciatura & & Licenciatura & & & & Máster \\
\hline$I I$ & Diplomatura & Máster & & Máster & & & \\
\hline 13 & Máster & Licenciatura & & & & & \\
\hline 14 & Licenciatura & & Máster & & Máster & & \\
\hline 15 & Diplomatura & & & & & & Diplomatura \\
\hline I6 & Máster & Licenciatura & & & & & \\
\hline$I 7$ & Licenciatura & & & & Licenciatura & & Máster \\
\hline 18 & Máster & & & & & Diplomatura & \\
\hline 19 & & Licenciatura & Máster & & & & \\
\hline 20 & Máster & & & & Licenciatura & & \\
\hline $2 I$ & Diplomatura & & & & & & \\
\hline 23 & Máster & & Diplomatura & & & & \\
\hline
\end{tabular}


La formación específica de los sujetos influye claramente en su actividad profesional. Por otra parte, si se atiende solamente al nivel de estudios mínimos (sin atender al área — traducción o ciencias-), la gran mayoría tiene una formación del nivel superior (máster); es decir, entre los sujetos de la encuesta son más numerosos aquellos que han estudiado hasta el nivel profesional más alto, el máster. Esto puede deberse a que, en muchos casos, los sujetos que respondieron a la encuesta accedieron a participar en el estudio tras participar en el máster específico de traducción de la Universitat Jaume I, lo que quizás daría un resultado sesgado respecto a la tendencia en el ámbito. En todo caso, esta variable puede servir para dar una idea sobre el nivel de formación de los informantes.

\subsection{Experiencia profesional}

En la encuesta se incluyó una pregunta sobre la cantidad de años de experiencia profesional.
TABLA 2. EXPERIENCIA PROFESIONAL

O. I33
O. 6788999
I. O
I. 5
$2 \cdot 002$
$2 \cdot 5$
$3 \cdot 4$
$4 \cdot 4$

La tabla 2 (diagrama de tallo y hojas) muestra que la gran mayoría de sujetos tiene una experiencia de menos de Io años, y solo tres de los I8, es decir, una sexta parte de los informantes, llevan más de 22 años de desempeño profesional.

En definitiva, puede decirse que los sujetos que han participado en el estudio tienen una experiencia suficiente traduciendo, aunque pocos pueden ser considerados expertos.

E1 gráfico 2 ordena el tipo de trabajo desempeñado durante los últimos seis meses

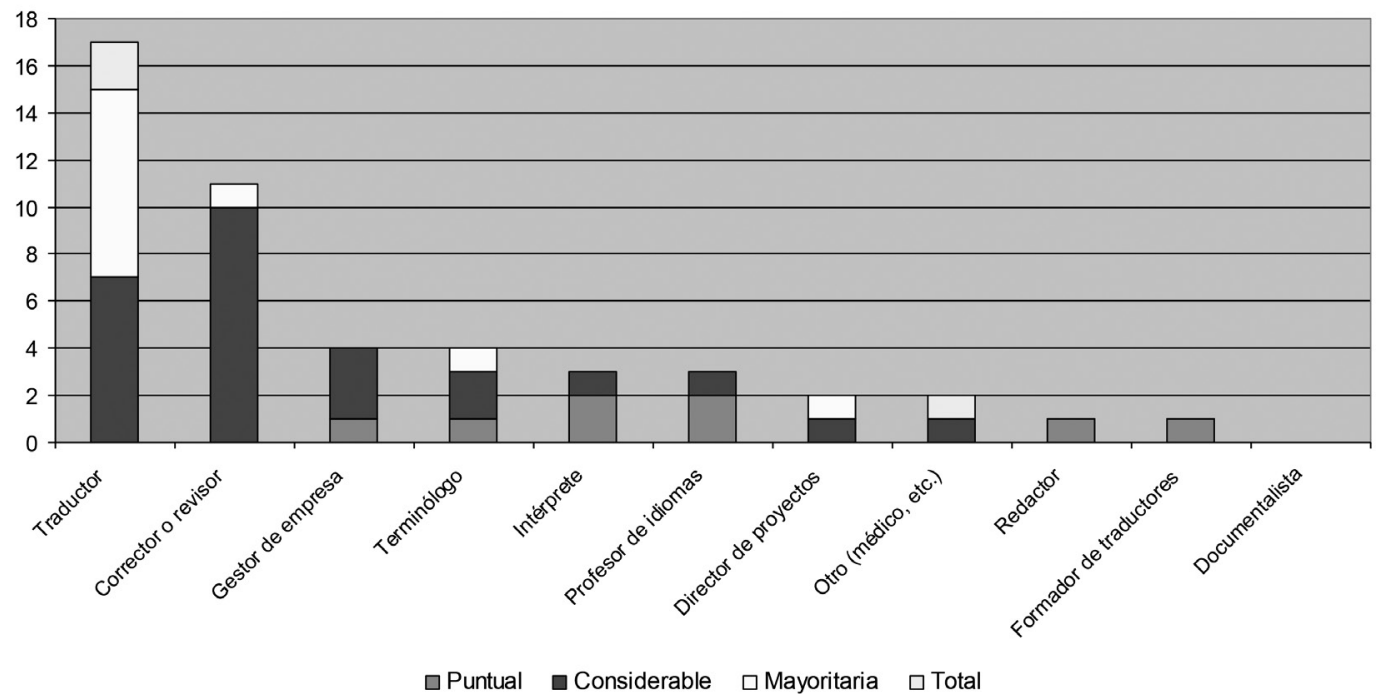

Gráfico 2. Tipo de tarea desempeñada durante los últimos seis meses 

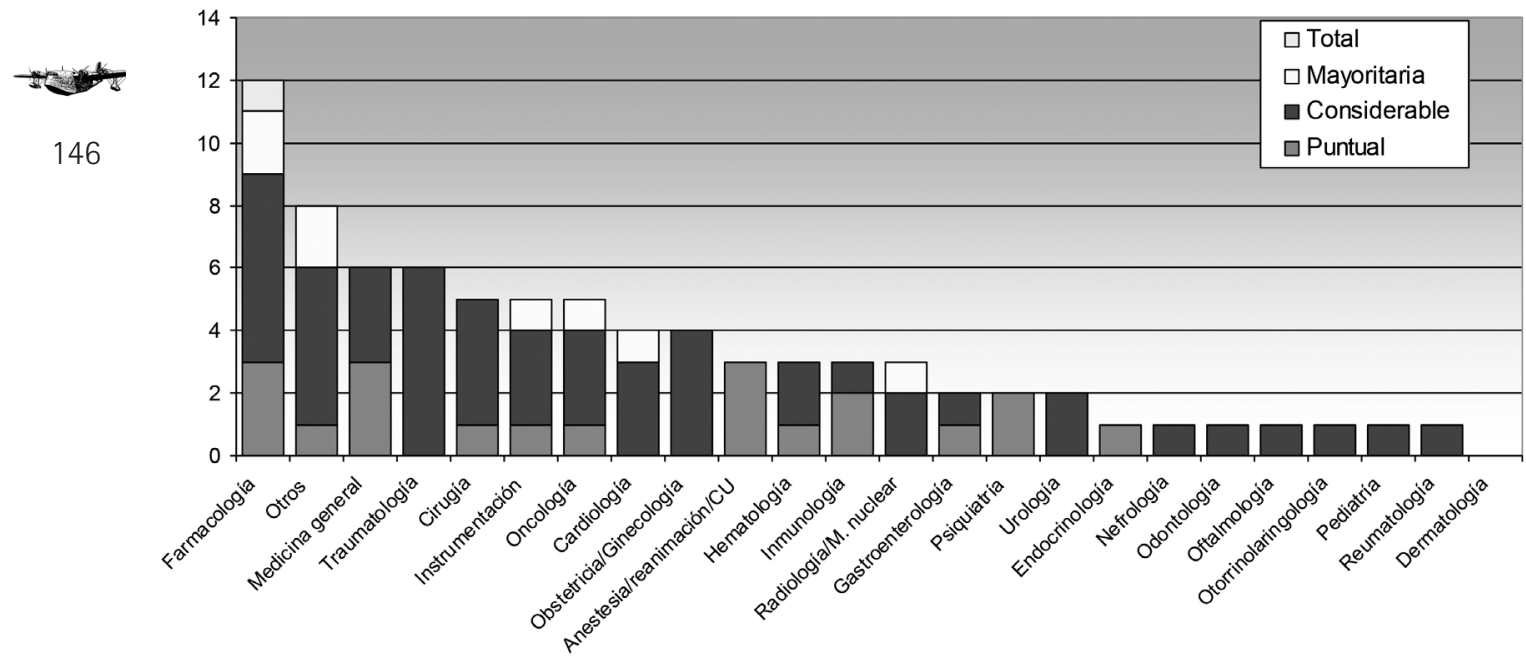

Gráfico 3. Ámbitos de las traducciones realizadas durante los últimos seis meses

por los sujetos, e incluye el porcentaje que dicho trabajo supone respecto de su volumen total.

Las tareas más desempeñadas, con diferencia, son las de traductor y corrector o revisor, seguidas de las de gestor y terminólogo. Nadie afirma haber realizado trabajos de documentalista, y de anecdóticas podrían denominarse las tareas de redactor y formador de traductores. La preeminencia de las labores de traductor se podría deber al hecho de que los sujetos del estudio fueron elegidos en una lista de traductores médicos. Llama la atención, de todos modos, la poca incidencia de los trabajos de interpretación (una tarea, quizás, muy especializada) y la supremacía de los trabajos de terminología sobre los de documentación que, en realidad, no los ha citado nadie. Esto podría deberse a que los sujetos consideran la documentación como una fase más de la traducción y no como una tarea aislada.

\section{Respuestas}

\section{I. Especialidades}

La primera pregunta del bloque A tenía que ver con el ámbito al que pertenecían los textos médicos que los sujetos decían haber traducido en los últimos seis meses. Como el anterior, el gráfico 3 muestra no solo el número de sujetos que menciona cada ámbito, sino también la dedicación que supone respecto del volumen de traducciones realizadas por cada informante.

Dos de cada tres sujetos dicen haber traducido textos del ámbito de la farmacología. Después de esta, las categorías más mencionadas han sido medicina general, traumatología, cirugía, instrumentación y oncología. Nadie ha traducido textos sobre dermatología, y anecdótica es la alusión a los textos de reumatología, pediatría, otorrinolaringología, oftalmología, odontología y nefrología.

Este gráfico podría servir para trazar un mapa sobre los temas más tratados en los textos médicos que necesitan una traducción. Es llamativo 
TABLA 3

OTROS ÁMBITOS DE LOS QUE SE HA TRADUCIDO

Enfermedades infecciosas

Análisis de laboratorio

Andrología

Asma

Bronconeumonología

Documentos de integración

Enfermería

Márketing de productos médicos

Materiales para pacientes

Medicina natural

Microbiología

Neurología

Nutrición

Parasitología

Prevención

Salud pública

Sanitarismo

Urgencias

$\mathrm{VIH}$

\section{Publicidad}

Textos legales

Traducción jurada

Cirugía maxilofacial y gastrointestinal que casi la mitad de sujetos dice haber traducido en los últimos 6 meses textos médicos de otros ámbitos que no son los que están en la lista, por lo que puede entenderse que no es exhaustiva. De todos modos, la encuesta ya lo preveía, y por eso se incluyó la siguiente pregunta, cuyas respuestas se resumen en la tabla 3. Solo ha habido un ámbito mencionado por más de una persona: el de las enfermedades infecciosas. El resto ha sido mencionado puntualmente. Los ámbitos incluidos en el fondo gris claro podrían considerarse errores de apreciación por parte de los sujetos, puesto que no se corresponden con textos específicos del ámbito médico, así como la categoría Cirugía maxilofacial y gastrointestinal, en gris oscuro, que sí que estaba incluida en la lista a la que hace referencia el gráfico 3 .

Es fácil advertir que las categorías de la tabla se sitúan a niveles distintos: desde las más generales, como Salud pública o Sanitarismo (que podrían considerarse sinónimas), prevención o enfermería; pasando por las relativas a especialidades médicas (Bronconeumología, Neurología,

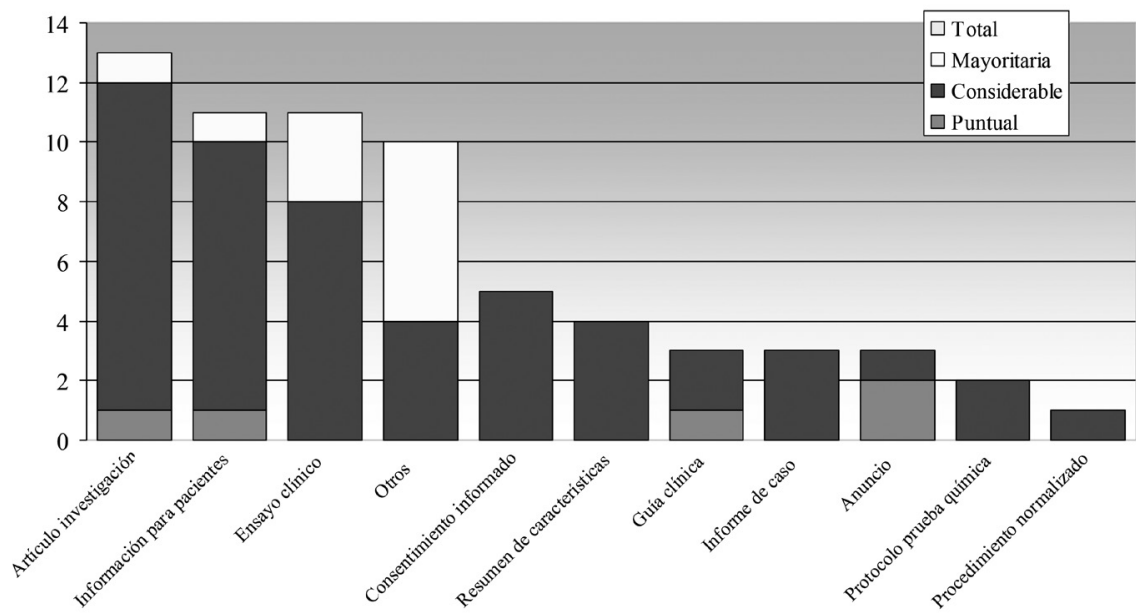

Gráfico 4. Géneros traducidos durante los últimos seis meses 
TABLA 4. OTROS GÉNEROS QUE SE HAN TRADUCIDO

Documentación para registro

2

Instrucción de uso de equipamiento

148

Manual de usuario
Página o sitio web

Software

Artículo para página web

Capítulo de libro

Carta para clientes

Cursos de capacitación para profesionales

Documentación administrativa y jurídica

Documentación interna

Encuesta médica

Especificación técnica

Folleto de presentación de medicamentos

Historia clínica

Libro

Manual de instrumentación

Medicina hiperbárica

Nota de aplicación

Oferta económica

Planificación

Póster (congresos)

Proyectos

Resumen

Revisión sistemática

SIDA

Técnica quirúrgica
En primer lugar está el artículo de investigación, citado por I3 sujetos. A continuación estaría la información para pacientes, el ensayo clínico y otros géneros médicos. Los menos citados son el procedimiento normalizado, el protocolo de prueba química, el anuncio, el informe de caso y la guía clínica. Lo primero que llama la atención es que todos los géneros textuales han sido citados, al menos, por un sujeto, lo que sugiere que dichos géneros están bien escogidos (existen, y se traducen, en el mercado). Es también curioso el hecho de que haya hasta 6 sujetos que afirmen traducir otro tipo de géneros de manera mayoritaria, es decir, que hay géneros importantes que no se han incluido en la lista, lo que no es sorprendente, puesto que se trataba únicamente de una primera aproximación ${ }^{7}$. Por último, a la luz de los resultados, parece que los destinatarios potenciales de las traducciones no son tanto los médicos o los hospitales como los pacientes e investigadores en medicina, pero esto se comprobará con la pregunta relativa al gráfico 5 .

La tabla 4 muestra los tipos de géneros médicos que los sujetos dicen haber traducido en los últimos meses pero que no se incluyen en la lista que ilustra el gráfico 4 . En 5 ocasiones coinciden más de un sujeto (2): documento de registro, instrucción de uso de equipamiento, manual de usuario, página web y software.

El gran número de géneros citados apunta a que la variedad de géneros médicos que necesitan traducción es realmente ingente. Entre los más nombrados hay dos casos de géneros

7 El grupo de investigación Gentt, a través de su unidad especializada en el ámbito médico, Medgentt, trabaja en los últimos años en el mapeado del ámbito a través de sus géneros más representativos, una primera aproximación del cual fue publicada en García Izquierdo (2009). Análisis como el que realizamos en este trabajo nos pueden ayudar a sistematizar de manera más rigurosa los géneros del ámbito. 


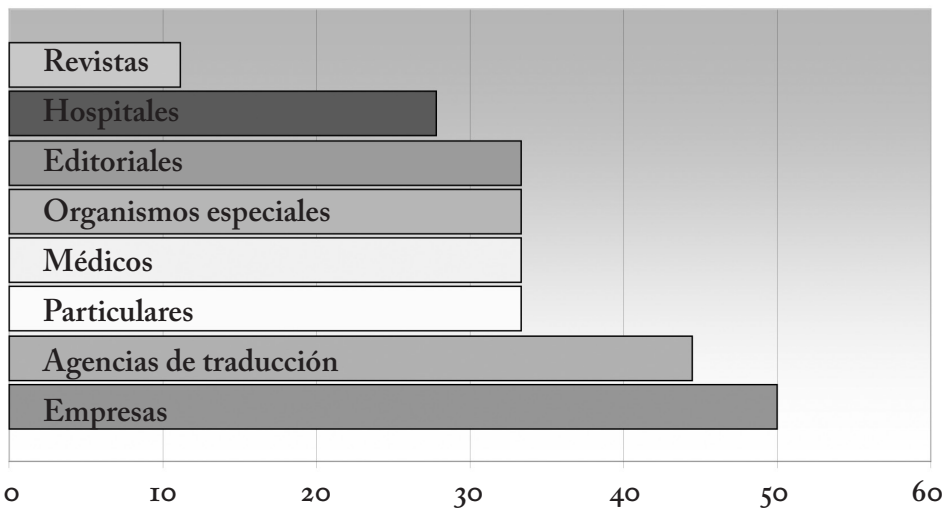

Gráfico 5. Géneros traducidos durante los últimos seis meses ligados a las nuevas tecnologías (software y páginas web) lo que indica una confusión entre el medio y el género. Por otra parte, la inclusión del SIDA como género vuelve a ser un error de apreciación por parte del informante, pues se confunden tema y género.

Los informantes debían contestar Sí o No a si traducían temas médicos para una serie de clientes. El gráfico 5 muestra que las empresas y las agencias de traducción son los destinatarios potenciales más citados por los sujetos; en el extremo opuesto estarían las revistas y los hospitales. Un tercio de los sujetos afirmaron traducir textos médicos para editoriales, organismos especiales, médicos y particulares. Por otra parte, cuatro traductores dijeron traducir textos para otro tipo de clientes: dos citaron laboratorios (uno, laboratorios médicos y el otro, farmacéuticos), otro dijo traducir textos típicos para la empresa para la que trabaja y el último citó varios ítems: enfermeras, trabajadores sociales, matronas y administración.

Parece que los traductores de textos médicos no suelen ser contratados directamente por clientes u hospitales para realizar las traducciones sino que es más habitual su contratación a través de agencias de traducción y empresas. Así, el mercado de la traducción médica no se diferencia mucho de otros mercados de traducción que también cuentan mayoritariamente con intermediarios. Creemos que, de confirmarse esta demanda, no sería descabellado plantearse la posibilidad de que los hospitales y otras instituciones públicas contrataran a traductores en plantilla. En cuanto a las respuestas al campo Otro, dos sujetos citaron laboratorios, lo cual nos lleva a considerar la necesidad de contar con esta opción en futuros estudios. Quien habló de la empresa en la que trabaja debería haber marcado la opción Empresas y, por último, el que citó a enfermeras, matronas, etc. tal vez debería haber marcado la opción Hospitales.

\subsection{Idiomas}

Un tipo especial de preguntas son las referidas a los idiomas de trabajo, principalmente por las implicaciones que tanto para las expectativas profesionales de los futuros traductores como para la organización académica de los escasos estudios existentes en la especialidad ${ }^{8}$ podrían acarrear.

8 Según muestran algunos estudios recientes $(\mathrm{Mu}-$ ñoz Miquel, 20I2), en España no existen apenas estudios específicos dedicados a la traducción médica; y, en el nivel de posgrado, destaca como única propuesta íntegramente en esta especialidad el Máster Universitario en Traducción médico-sanitaria de la Universitat Jaume I de Castellón. 
150

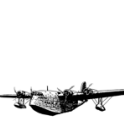

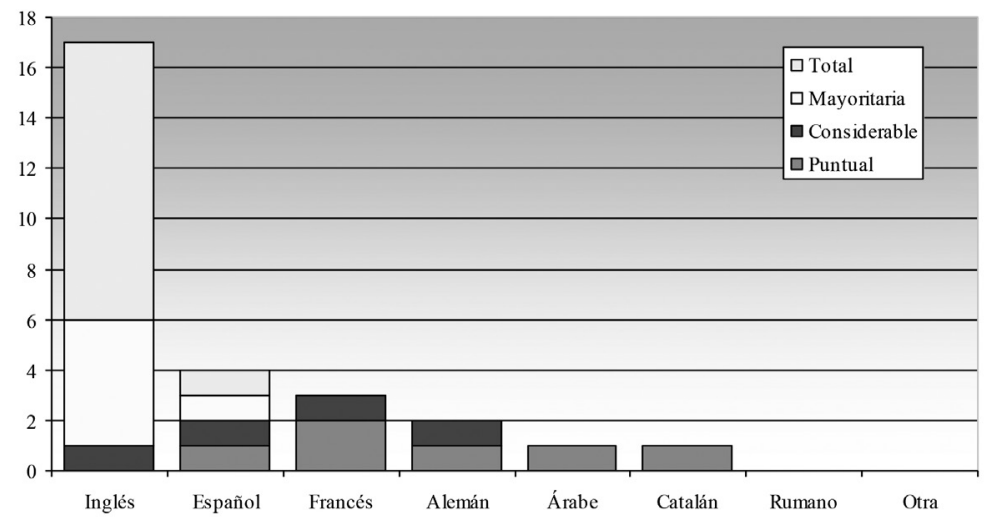

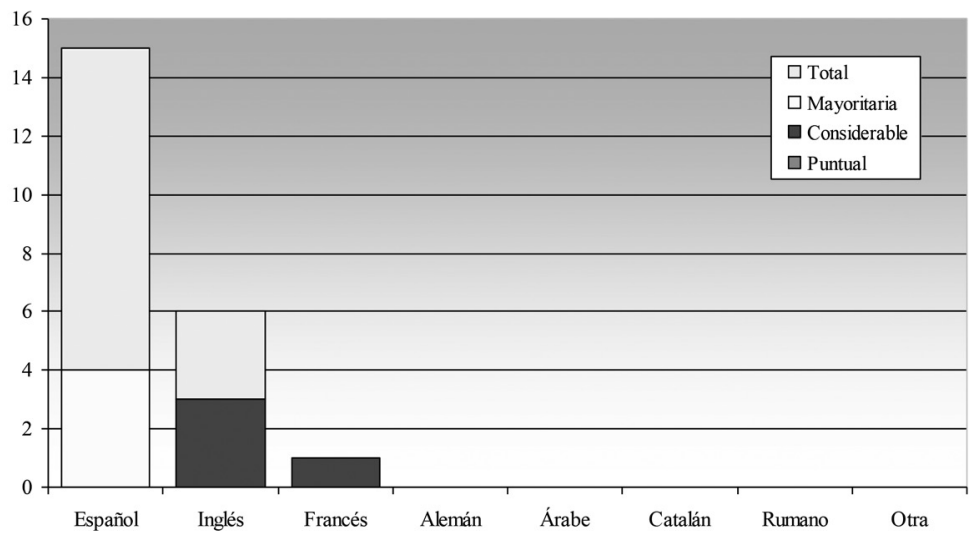

Gráfico 6. Lenguas desde las que traducen
Gráfico 7. Lenguas hacia las que traducen
El gráfico 6 ordena las lenguas originales de más a menos traducidas. Además del número total de sujetos que han citado cada idioma, se incluye información sobre qué porcentaje de textos supone cada idioma en la producción de cada sujeto (puntual: < $10 \%$; considerable: Io-50 \%; mayoritaria: 5I-99 \% y total: Ioo \%).

La supremacía del inglés es evidente, no solo en el número de sujetos que la han seleccionado, sino en el hecho de que in de ellos solo traducen del inglés. Llama la atención también que se realiza más traducción desde el español que desde el francés, el alemán, el árabe o el rumano, lengua que no se ha citado ni una sola vez ${ }^{9}$. Por otra parte, el hecho de que nadie haya nombrado otra nombre habla a favor de la exhaustividad e idoneidad de la lista de categorías elegidas.

La información del gráfico anterior se completa con la resumida en el siguiente gráfico 7 , en el que se muestran ordenadas de mayor a menor las lenguas a las que traducen los sujetos incluidos en el estudio. El español es el idioma

$9 \quad$ La existencia de importantes núcleos de población rumana en los últimos años en algunas provincias españolas nos llevó a considerar su inclusión en la encuesta. 


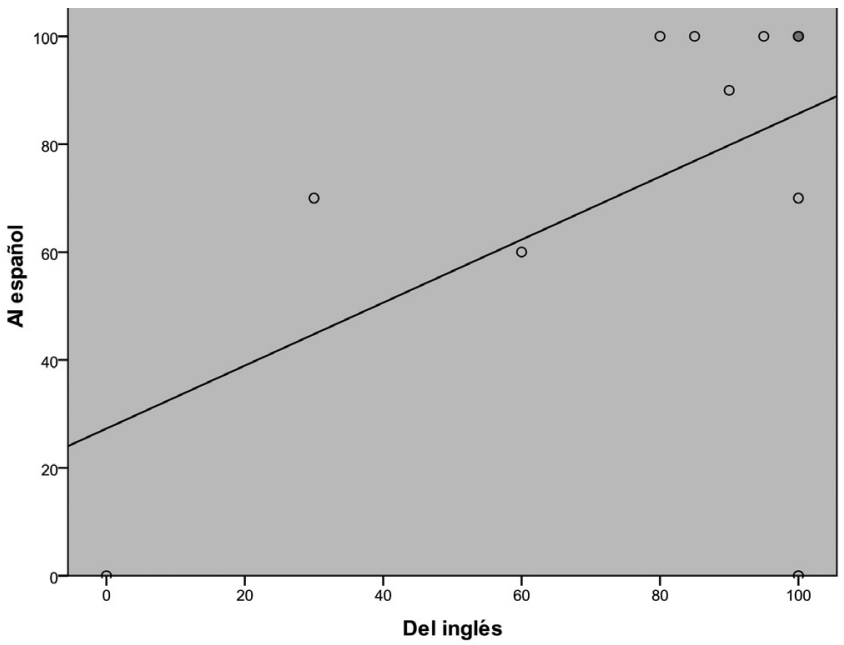

Gráfico 8. Relación entre quienes traducen del inglés y quienes traducen al español más utilizado, con diferencia, seguido del inglés $\mathrm{y}$, puntualmente, por el francés.

Se confirma la traducción directa al español como la actividad más realizada por los traductores médicos incluidos en el estudio, así como la supremacía de la traducción inversa al inglés sobre la realizada a otros idiomas y sobre la traducción directa al catalán, que no recibe ni una sola mención, (como ocurre con el alemán, el árabe o el rumano). Como en el caso anterior, la lista parece exhaustiva, por cuanto ningún sujeto marcó la opción Otras.

El gráfico ocho apunta a una correlación entre los que traducen del inglés y los que lo hacen al español: cuanto más volumen de trabajo se hace del inglés, más se traduce al español. Así, hay algunos (hasta ir sujetos, punto oscuro en el gráfico) que solo ejecutan traducción directa inglés-español ${ }^{10}$.

ro Por último, cabría considerar como valor atípico al sujeto 23, que no debió entender las preguntas correspondientes a los gráficos 6 y 7 , pues respondió que en los últimos 6 meses todas sus traducciones habían sido desde el inglés hacia el inglés.

\subsection{Corpus}

La primera pregunta del bloque B versaba sobre los recursos de tipo documental que los sujetos utilizan para traducir temas médicos. En el gráfico 9 están ordenados de más a menos utilizados, según las respuestas obtenidas para el uso de los recursos en formato electrónico pues, como puede apreciarse, es el más utilizado en todos los casos (solo los diccionarios bilingües son utilizados al mismo nivel en papel y en formato electrónico).

Los diccionarios monolingües son el recurso más utilizado en formato electrónico, seguido de las comunidades virtuales de expertos, las bases de datos terminológicas y los diccionarios bilingües. En formato papel, los diccionarios (monolingües y bilingües) son el recurso más utilizado, seguido de los manuales y, a mucha distancia, los tesauros. Es destacable que los expertos (traductores o no) solo sean consultados en línea (por medio de los foros) y que las bases de datos, los corpus y los tesauros se pueden considerar, hasta cierto punto, herramientas eminentemente electrónicas, al menos en lo que respecta a su uso reconocido. 
Diccionarios monolingües

Comunidades virtuales de expertos

Bases de datos terminológicas

Diccionarios bilingües

Manuales y obras conceptuales

Corpus o compilaciones de textos

Comunidades virtuales de traductores

Tesauros
Memorias de traducción

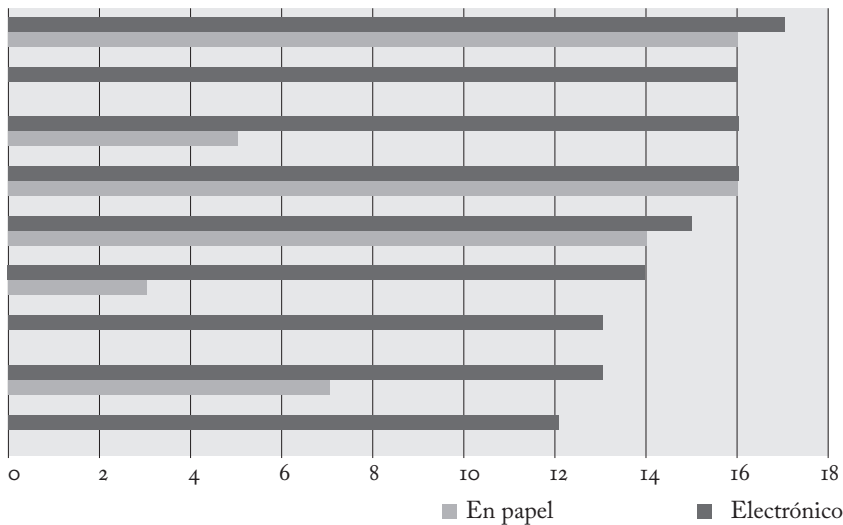

Gráfico 9. Recursos documentales utilizados, en papel y electrónicos

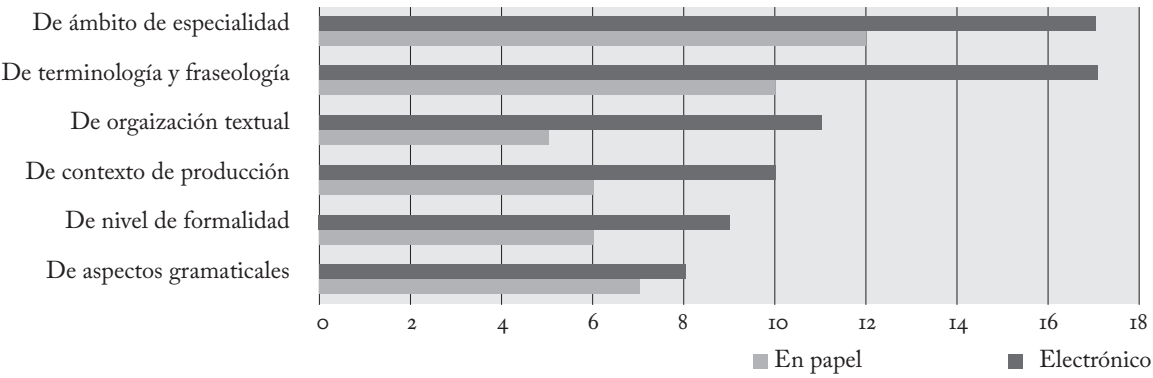

Gráfico Io. Razones para las búsquedas documentales, en papel y electrónicas

En otra pregunta de este bloque se abordaba la cuestión del tipo de información que sería deseable encontrar en los corpus. En el gráfico ro se han ordenado las categorías según el número de respuestas respecto al campo Electrónicos por ser, en general y de nuevo, el más seleccionado. Así, se necesitan corpus electrónicos principalmente para consultar el ámbito de especialidad, la terminología y la fraseología. Las categorías menos citadas en este formato son los aspectos textuales y el nivel de formalidad. Los corpus en papel serían utilizados, según los sujetos, también para los dos usos principales de los corpus electrónicos (termino- logía y fraseología), aunque los aspectos gramaticales estarían en este formato por encima del nivel de formalidad, el contexto de producción y la organización textual.

Independientemente del formato del corpus, los encuestados tienen clara cuál sería la información que querrían encontrar en ellos: la relativa al ámbito de especialidad (es decir, conceptual) y la terminológica/fraseológica. La mayor relevancia de los aspectos gramaticales en los corpus en papel podría deberse a una cuestión de tradición. Por otra parte, el mayor peso que las dudas de organización textual tienen en los corpus electrónicos puede deberse a 
TABLA 5. OTRAS OBRAS DE CONSULTA DESTACABLES

\begin{tabular}{ll}
\hline Diario crítico de dudas inglés-español de medicina & 8 \\
\hline Stedman bilingüe & 2 \\
\hline Diccionario de uso del español & I \\
Diccionario médico (Dox-Melloni-Eisner) & I \\
Dorland Diccionario enciclopédico ilustrado & \\
$\quad$ de medicina & I \\
DRAE en línea & I \\
Farmacología humana (Flórez) & I \\
Manuales especializados & I \\
Medicina interna (Farreras y Rozman) & I \\
Merriam Webster & I \\
NCBi Diccionario terminológico & \\
$\quad$ de las Ciencias Médicas & I \\
PubMed & I \\
\hline
\end{tabular}

que el formato electrónico hace más fácil el análisis de la organización textual, especialmente en los textos de un tamaño considerable.

A continuación, se hizo a los sujetos una pregunta opcional sobre otras obras de consulta destacables o de especial utilidad. Hasta 8 sujetos citaron el Diccionario crítico de dudas inglés-español de medicina de Fernando Navarro (tabla 5). Otros diccionarios mencionados, aunque en mucha menor medida, han sido los médicos (Dox, Melloni, Eisner, Stedman, NCBI y Merriam Webster) y los generales (de uso, DRAE). Por otra parte, se han citado páginas web (PubMed) y manuales generales y específicos (farmacología y medicina interna).

En la pregunta siguiente, se indagaba acerca de la finalidad de las consultas terminológicas realizadas por los sujetos, concretamente si las realizaban para encontrar el término concreto en la lengua meta o para saber el significado. Cada posibilidad tenía tres opciones: nunca, a menudo y siempre. El gráfico II muestra que ninguno ha respondido Nunca a ninguna de las dos posibilidades. Dos tercios de los encuesta- $\square$ Siempre $\square$ A menudo

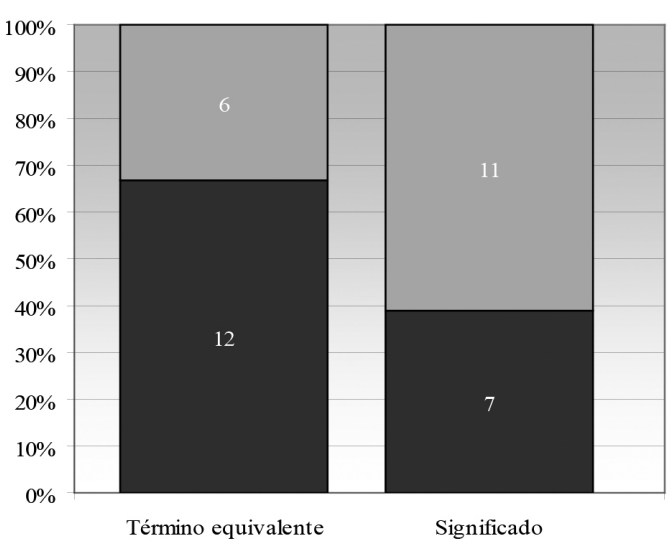

Gráfico Ir. Finalidad de las consultas terminológicas

dos dicen utilizar el corpus siempre para encontrar un término equivalente en la lengua a la que traduce y aproximadamente un tercio dice usarlo también siempre para saber el significado de un término. En ambos casos, el resto de sujetos reconocen utilizar un corpus a menudo para resolver dichas cuestiones.

Para los traductores médicos, las consultas terminológicas son casi siempre para resolver o comprobar términos equivalentes en la lengua a la que se traduce, aunque, a raíz de los datos, podemos deducir que está muy extendido el uso del corpus para saber el significado de ciertos términos especializados.

Los corpus médicos que los sujetos ya conocen son mejorables. La tabla 6 resume el tipo de información que los traductores médicos echan de menos en este tipo de compilaciones.

Solo uno de los sujetos dijo que los corpus eran buenos y completos, es decir, no criticó ningún aspecto de los corpus porque los encontraba ya lo suficientemente útiles. Otros 5 sujetos prescindieron de la crítica, pero por motivos diversos: no conoce ninguno lo suficientemente 
TABLA 6.

INFORMACIÓN QUE QUERRÍAN ENCONTRAR EN LOS CORPUS

\section{Críticas negativas}

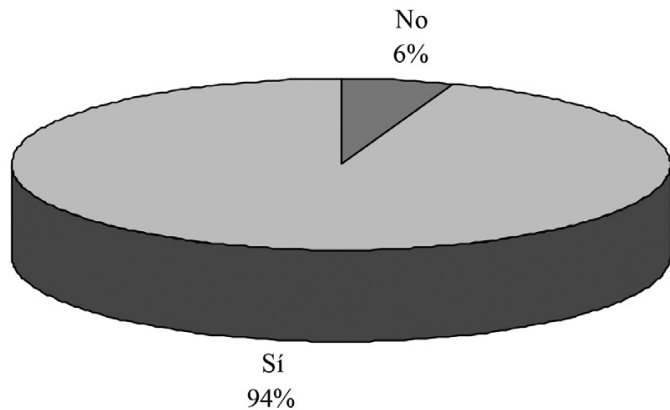

Gráfico I2. Utilidad de un corpus médico especializado

matices semánticos, uso, vocabulario específico, abreviaturas y, en un caso, sobre la presentación de la información o estructura, difícil de entender — según el informante- para los médicos no familiarizados con estas herramientas.

En resumen, las críticas y mejoras son variadas y responden, probablemente, a los ejemplos de corpus a los que han tenido acceso los sujetos, que adolecen de distintas cosas. Esto podría explicar la propuesta de que algunos corpus incorporen información sobre colocaciones o ejemplos (información, como es sabido, ya existente en gran variedad de corpus). Es, por último, llamativo que hasta 5 sujetos digan no conocer o no utilizar los corpus, por cuanto en los últimos años esta herramienta ha demostrado ser muy valiosa para la práctica profesional. Sirva para confirmar este dato la última pregunta de este bloque: ¿Le resultaría útil un corpus médico del que pudiera extraer la información señalada en las preguntas anteriores? El gráfico I2 muestra que solo uno de los participantes en el estudio contestó que No.

De manera casi unánime, pues, se considera el corpus una herramienta valiosa. cuenta que la mayoría de ellos, como hemos apuntado antes, solo traducen del inglés al español. 
TABLA 7. ÁMBITOS SEGÚN PERICIA

\begin{tabular}{|c|c|c|c|c|}
\hline & \multicolumn{2}{|c|}{$<8$ años de pericia } & \multicolumn{2}{|c|}{$\geq 8$ años de pericia } \\
\hline & Recuento & $\%$ del $\mathrm{N}$ total & Recuento & $\%$ del $\mathrm{N}$ total \\
\hline Farmacología & 2 & 40,0 & IO & 76,9 \\
\hline Otros & 2 & 40,0 & 6 & 46,2 \\
\hline Medicina general & I & 20,0 & 5 & 38,5 \\
\hline Traumatología & I & 20,0 & 5 & 38,5 \\
\hline Cirugía & I & 20,0 & 4 & 30,8 \\
\hline Instrumentación & I & 20,0 & 3 & $23, \mathrm{I}$ \\
\hline Oncología & 2 & 40,0 & 3 & $23, \mathrm{I}$ \\
\hline Cardiología & I & 20,0 & 3 & $23, \mathrm{I}$ \\
\hline Obstetricia/Ginecología & I & 20,0 & 3 & $23, \mathrm{I}$ \\
\hline Anestesia/reanimación/cu & 2 & 40,0 & I & 7,7 \\
\hline Hematología & I & 20,0 & I & 7,7 \\
\hline Inmunología & 2 & 40,0 & I & 7,7 \\
\hline Radiología/M. nuclear & I & 20,0 & 2 & 15,4 \\
\hline Gastroenterología & ० & 0,0 & 2 & $\mathrm{I} 5,4$ \\
\hline Psiquiatría & o & 0,0 & 2 & 15,4 \\
\hline Urología & I & 20,0 & I & 7,7 \\
\hline Endocrinología & I & 20,0 & o & 0,0 \\
\hline Nefrología & o & ০,० & I & 7,7 \\
\hline Odontología & ० & ০,० & I & 7,7 \\
\hline Oftalmología & I & 20,0 & ○ & 0,0 \\
\hline Otorrinolaringología & o & $\circ, 0$ & I & 7,7 \\
\hline Pediatría & ○ & ०,० & I & 7,7 \\
\hline Reumatología & ○ & ०,० & I & 7,7 \\
\hline
\end{tabular}

\subsection{Pericia}

Tras realizar el análisis de las preguntas contenidas en los principales bloques de la encuesta, podría plantearse si algunos resultados no podrían estar asociados a un determinado nivel de experiencia. Por ello, hemos dividido a los sujetos, por mor del análisis, en dos grupos según su nivel de pericia: quienes tienen siete o menos de siete años de experiencia y quienes tienen más de siete años de experiencia (véase $\S$ I.3). El primer grupo contendría un total de cinco traductores (el $28 \%$ ) y el segundo, trece.

Por cuanto esta variable puede resultar muy informativa para perfilar a los sujetos del estudio, en esta sección se ha resuelto cruzar esta variable con el resto de parámetros de la encuesta. Así, en la tabla 7 las filas muestran los ámbitos ordenados según su orden de menciones, mientras que las columnas exponen el recuento y el porcentaje de sujetos que, dentro de cada grupo según pericia, representan según el total de sujetos de dicho grupo.

Tomando cada grupo de manera aislada, los menos expertos trabajan en una menor variedad de ámbitos; así, no traducen textos de los siguientes ámbitos: Gastroenterología, Psiquiatría, Nefrología, Odontología, Otorrinolaringología, Pediatría y Reumatología. En cuanto 
Tabla 8. Géneros traducidos SEgún PERICIA

\begin{tabular}{|c|c|c|c|c|c|}
\hline \multirow{2}{*}{156} & & \multicolumn{2}{|c|}{$<8$ años de pericia } & \multicolumn{2}{|c|}{$\geq 8$ años de pericia } \\
\hline & & Recuento & $\%$ del $\mathrm{N}$ total & Recuento & $\%$ del $\mathrm{N}$ total \\
\hline & Artículo investigación & 4 & 80,0 & IO & 76,9 \\
\hline & Info. para pacientes & 3 & 60,0 & 8 & $6 \mathrm{I}, 5$ \\
\hline & Ensayo clínico & 3 & 60,0 & 8 & $6 \mathrm{I}, 5$ \\
\hline & Otros & 2 & 40,0 & 8 & $6 \mathrm{I}, 5$ \\
\hline & Consentimiento informado & I & 20,0 & 4 & 30,8 \\
\hline & Resumen características & I & 20,0 & 3 & $23, \mathrm{I}$ \\
\hline & Guía clínica & o & 0,0 & 3 & $23, \mathrm{I}$ \\
\hline & Informe de caso & I & 20,0 & 2 & $\mathrm{I} 5,4$ \\
\hline & Anuncio & I & 20,0 & 2 & 15,4 \\
\hline & Protocolo prueba química & o & 0,0 & 2 & 15,4 \\
\hline & Procedimiento normalizado & ० & 0,0 & I & 7,7 \\
\hline
\end{tabular}

a los cambios de orden respecto de la tendencia general, se aprecia un mayor peso de los ámbitos de la Anestesia, la Oncología y la Inmunología; no obstante, solo hay cinco traductores poco expertos, y las diferencias son grandes en porcentajes pero pequeñas en número de traductores. Por otra parte, los únicos ámbitos que no han trabajado los traductores más expertos son la Endocrinología y Oftalmología. No presentan muchos cambios de orden respecto de la tendencia, si bien dan más peso a los ámbitos de la Radiología, la Gastroenterología y la Psiquiatría. Si se comparan los dos grupos, se ve que hay ámbitos que solo son traducidos por expertos o por novatos, que los más peritos afirman traducir textos de una mayor variedad de ámbitos y que el porcentaje de sujetos que, dentro de cada grupo, dice traducir textos de los distintos ámbitos suele ser también mayor en los más expertos que en los menos, como era esperable.

Al cruzar los datos relacionados con los géneros con los de años de experiencia puede observarse qué ámbitos son los más usuales en cada etapa de la pericia. La tabla 8 muestra el porcentaje de sujetos que, dentro de cada grupo, se ha ocupado alguna vez de los distintos géneros.

Dentro del grupo de los menos expertos, la guía clínica no tiene tanto peso como el que debiera, según la tendencia central. En cambio, en el grupo de los traductores más expertos, los distintos géneros tienen la importancia que dicta la tendencia general. En casi todos los casos, el subgrupo de traductores expertos muestra un porcentaje mayor de géneros mencionados. Excepciones, por tanto, son las ocasiones en las que el porcentaje es mayor en los menos expertos: el artículo de investigación, el resumen de características, el informe de caso y el anuncio. Una posible explicación para esto es que los novatos (cuyas tarifas, además, serán más bajas) se atreven a traducirlos por considerar que se trata de géneros menos comprometidos en cuanto a su repercusión. De todos modos, las diferencias son poco significativas. Finalmente, mientras que todos los géneros incluidos en la encuesta han sido citados por alguno de los 
TABLA 9. CLIENTES POTENCIALES SEGÚN PERICIA

\begin{tabular}{lcccc}
\hline & \multicolumn{2}{c}{$<8$ años de pericia } & \multicolumn{2}{c}{$\geq 8$ años de pericia } \\
\cline { 2 - 5 } & Recuento & \% del N total & Recuento & \% del N total \\
\hline Artículo investigación & 4 & 80,0 & Io & 76,9 \\
Empresas & I & 20,0 & 8 & $6 \mathrm{I}, 5$ \\
Agencias de traducción & 2 & 40,0 & 6 & 46,2 \\
Particulares & 2 & 40,0 & 4 & 30,8 \\
Médicos & 2 & 40,0 & 4 & 30,8 \\
Organismos oficiales & 0 & 0,0 & 6 & 46,2 \\
Editoriales & I & 20,0 & 5 & 38,5 \\
Hospitales & 2 & 40,0 & 3 & $23, \mathrm{I}$ \\
Revistas & 0 & 0,0 & 2 & 15,4 \\
\hline
\end{tabular}

traductores expertos, hay dos géneros que nunca han sido citados por los menos expertos: el protocolo de prueba química y el procedimiento normalizado. Esto sugiere que hay géneros más complejos, tanto por su difícil acceso y configuración, como por las repercusiones que su contenido puede tener en el sistema de salud y, por tanto, por su especial dificultad, solo los sujetos más peritos y con mejores contactos están en disposición de realizar su traducción.

Una operación análoga se ha realizado con los clientes potenciales. Así, la tabla 9 muestra el porcentaje que, dentro de cada nivel de pericia, representa el número de sujetos que han trabajado para las categorías de clientes contempladas.

En el grupo de los menos expertos, los hospitales tienen más peso del que dicta la tendencia general y las empresas y los organismos oficiales, menos. Mientras tanto, en el grupo de los más expertos destacan los organismos oficiales, que tiene mayor importancia de la que sería esperable según la tendencia central. Cabría señalar que hay dos clientes que nunca han encargado traducciones a estos sujetos: los organismos oficiales y las revistas.

En la tabla ro se muestran los recursos utilizados por los dos grupos de sujetos, tanto en formato digital o virtual, como en papel.

Empecemos por las dos primeras columnas. En el grupo de menos expertos hay una categoría con menor relevancia de la que se esperaría (las comunidades de expertos) y otra, con mayor peso (tesauros). En los más expertos el escalafón es perfecto en relación a la tendencia general. En cuanto al uso de fuentes en papel, la diferencia más notoria se da en la mención a los tesauros: muy mayoritaria entre los menos expertos, y apenas anecdótica entre los más expertos. Parece, pues, característico de los traductores médicos menos expertos el uso de los tesauros, sea en formato electrónico, o en papel. Una posible explicación a esto es que a los novatos, recién licenciados en su mayoría, se les ha animado desde las aulas a que utilizaran este tipo de recursos que luego, cuando son más expertos en la materia, deciden abandonar, bien porque han descubierto otras herramientas que 
TABLA IO. RECURSOS DOCUMENTALES UTILIZADOS SEGÚN PERICIA

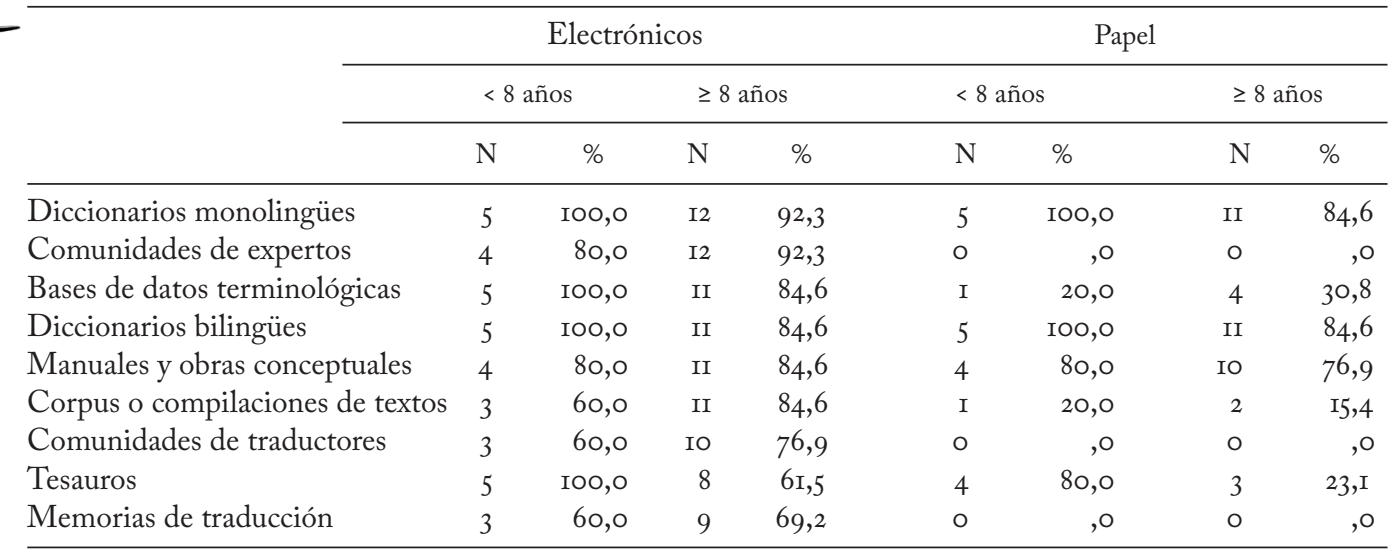

TABLA II. BÚSQUEDAS TERMINOLÓGICAS SEGÚN PERICIA

\begin{tabular}{|c|c|c|c|c|c|c|c|c|}
\hline & \multicolumn{4}{|c|}{ Electrónicos } & \multicolumn{4}{|c|}{ Papel } \\
\hline & \multicolumn{2}{|c|}{$<8$ años } & \multicolumn{2}{|c|}{$\geq 8$ años } & \multicolumn{2}{|c|}{$<8$ años } & \multicolumn{2}{|c|}{$\geq 8$ años } \\
\hline & $\mathrm{N}$ & $\%$ & $\mathrm{~N}$ & $\%$ & $\mathrm{~N}$ & $\%$ & $\mathrm{~N}$ & $\%$ \\
\hline De ámbito de especialidad & 5 & IOO,O & $\mathrm{I} 2$ & 92,3 & 3 & 60,0 & 9 & 69,2 \\
\hline De terminología y fraseología & 5 & IOO,O & $\mathrm{I} 2$ & 92,3 & 2 & 40,0 & 8 & $6 \mathrm{I}, 5$ \\
\hline De organización textual & 3 & 60,0 & 8 & $6 \mathrm{I}, 5$ & 2 & 40,0 & 3 & $23, \mathrm{I}$ \\
\hline De contexto de producción & 4 & 80,0 & 6 & 46,2 & 2 & 40,0 & 4 & 30,8 \\
\hline De nivel de formalidad & 4 & 80,0 & 5 & 38,5 & 2 & 40,0 & 4 & 30,8 \\
\hline De aspectos gramaticales & 3 & 60,0 & 5 & $3^{8,5}$ & 3 & 60,0 & 4 & 30,8 \\
\hline
\end{tabular}

les resultan más útiles, bien porque ya no los necesitan, por haber llegado a un dominio suficiente en la terminología típica de su ámbito.

Por otra parte, también se han buscado diferencias en la información que los dos grupos de sujetos desean encontrar en los corpus, para lo que se ha confeccionado la tabla II.

Para las categorías virtuales, el único cambio dentro del grupo de los menos expertos está en el de organización textual, menos importante de lo que sería esperable; en el de los más expertos, el escalafón es completamente normal. Llama la atención también ese roo \% de sujetos menos expertos que dicen buscar información sobre terminología y ámbito de especialidad. Para las categorías en papel, los menos expertos inciden más de lo esperable, por tendencia, en los aspectos gramaticales; mientras que los menos expertos buscan en fuentes impresas menos información que la media sobre organización textual. Comparativamente, los más expertos suelen mostrar porcentajes menores, quizás por su nivel de pericia.

Por último, el gráfico I3 divide a los traduc- 


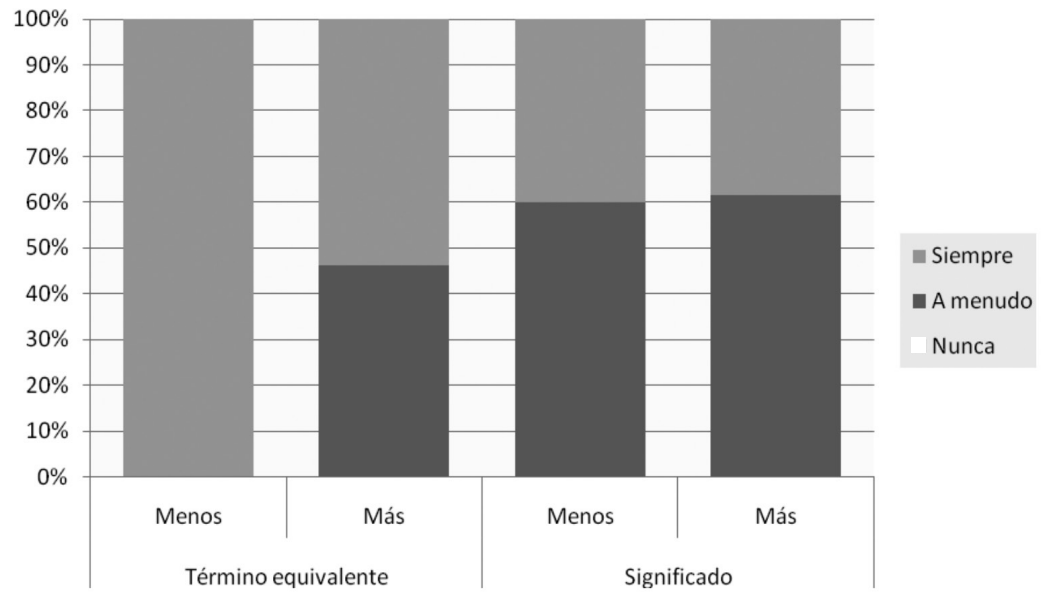

Gráfico 13. Finalidad de las consultas terminológicas según pericia tores en más y menos expertos y, dentro de ellos, muestra el porcentaje de sujetos que dicen emplear las consultas terminológicas bien para buscar el término equivalente (primeras dos columnas), bien para aprender el significado (siguientes dos columnas).

No se aprecian diferencias en la segunda categoría: los dos grupos de sujetos afirman utilizar de una manera similar las consultas terminológicas cuando se trata de buscar el significado de un término. Así, esta actividad no está relacionada con el nivel de experiencia del traductor. No obstante, para la otra categoría sí se aprecia una diferencia considerable: todos los menos expertos afirman realizar siempre búsquedas terminológicas para hallar el término equivalente; entre los expertos solo la mitad eligen la opción siempre. Parece, pues, que entre los traductores más expertos resulta menos obligado el realizar consultas terminológicas para hallar el término equivalente, probablemente porque los años de experiencia les han proporcionado mayor seguridad y un mejor conocimiento de la forma y el contenido, en la lengua meta, de los géneros que traducen. Este resultado concuerda con el antes apuntado sobre el menor uso que estos sujetos hacen de los tesauros y apunta, en definitiva, a una menor dependencia, entre los más expertos, de las fuentes de documentación especializada.

\section{CONCLUSIONES}

A pesar de ser uno de los ámbitos más presentes en la profesión, a la traducción médica no se le ha prestado la suficiente atención desde los entornos investigadores. Esto es especialmente grave en el caso de la documentación, pues quienes suelen ocuparse de esta actividad conforman una comunidad más heterogénea que la de otros profesionales de la traducción.

Por medio de encuestas, hemos querido conocer cómo trabajan y qué opinión tienen sobre ello los profesionales de la traducción médica en España, haciendo especial hincapié en los procesos de documentación. Hemos contado para ello con dieciocho sujetos procedentes de tres grupos específicos de traductores médicos.

El participante medio de la encuesta es una mujer de entre treinta y cuarenta años, experta 
en Traducción y alguna rama de la Ciencia y con estudios de máster. Tiene una experiencia profesional de entre 6 y 9 años; recientemente, ha ejercido labores, sobre todo, de traductora o correctora/revisora en diversos ámbitos, aunque destacan su trabajo en la rama de Farmacología; y los géneros que más ha traducido son el artículo de investigación, la información para pacientes y el ensayo clínico. Su cliente tipo es una empresa o agencia de traducción; su lengua origen, el inglés y su lengua meta, el español.

Mención aparte merece el uso que el encuestado medio hace de los recursos documentales, que necesitan, sobre todo, para consultar dudas terminológicas, fraseológicas o relativas al ámbito de especialidad. Destaca la utilización de diccionarios, bases de datos y comunidades virtuales de expertos en la materia que tienen que traducir; y estas consultas están enfocadas especialmente a la fase de producción del texto meta. Reconocen la utilidad de los corpus especializados sobre los que vierten críticas constructivas de muy diversa índole.

Para completar el perfil del encuestado medio se ha distinguido entre traductores novatos y traductores expertos y se ha descubierto que estos últimos se caracterizan por: trabajar en un abanico más amplio de ámbitos especializados y géneros textuales; llegar hasta clientes inaccesibles a los menos expertos; no utilizar tesauros; y realizar menos consultas documentales, sobre todo en la identificación de términos equivalentes.

Más allá del perfil medio del informante, del análisis precedente se deducen varias conclusiones. La primera tiene que ver con la significativa heterogeneidad de los profesionales analizados: traducen una gran variedad de géneros textuales relativos a distintos ámbitos de especialidad, lo que indica un campo rico y amplio, susceptible en definitiva de ser abordado con ayudas docu- mentales de diversos tipos. Esta heterogeneidad solo se rompe, en parte, cuando se comprueban las vías de contratación, que casi siempre pasan por agencias y empresas de traducción, esto es, por intermediarios ${ }^{12}$.

Por otra parte, el análisis de los recursos documentales ha confirmado la supremacía de las consultas en línea y dos hechos que nos han resultado curiosos: la consulta documental se utiliza más en la fase de traducción que en la fase de comprensión y los corpus de textos especializados gozan de una buena reputación, a pesar de que en muchos casos los informantes demostraron no conocer bien las herramientas y posibilidades que estos ofrecen. La importancia de las consultas terminológicas enfocadas a la elección apropiada de la terminología viene dada, con toda seguridad, a la importancia que esta cuestión tiene en la traducción científica en general y en la médica, en particular. No basta con entender el texto y proponer una versión más o menos aproximada en la lengua meta, el traductor médico se esfuerza por utilizar el término más adecuado en cada caso, lo cual puede conseguir con herramientas de gestión de corpus como esas que tanto valora y tan poco conoce. Quizás esta infrautilización tenga que ver, también, con el hecho de que no conozca corpus especializados en su propio campo, lo cual, como explicaremos ahora, tiene solución.

Para acabar con las conclusiones, añadiremos que el estudio de las diferencias observadas en relación con la pericia ha puesto de manifiesto cómo los dos grupos analizados necesitan y agradecen la existencia de recursos documentales enfocados a la traducción médica, si bien esta

I2 Esto contrasta con profesionales que trabajan en otros ámbitos; por ejemplo, en un artículo anterior, descubrimos (García-Izquierdo y Conde, 20I2: I48) que entre los traductores jurídicos era más habitual la contratación directa con el cliente, sin intermediarios de ningún tipo. 
necesidad se hace más acuciante en el grupo de traductores menos expertos, quienes muestran un uso más habitual de determinados recursos y una mayor inseguridad, como demuestra el hecho de que realicen consultas terminológicas con mayor frecuencia que los integrantes del grupo de expertos.

Entendemos que un estudio de este tipo tiene sus limitaciones; para empezar, la muestra podría no ser totalmente representativa y estar sesgada por ser la mayoría de los informantes alumnos o antiguos alumnos del Máster de Traducción Médico-Sanitaria de la Universitat Jaume I. A pesar de ello, creemos que los resultados son informativos y ofrecen un panorama muy completo e ilustrativo de la profesión del traductor médico en España. No abundan los estudios cuantitativos sobre este perfil profesional y, en ese sentido, creemos que venimos a rellenar un hueco, al menos iniciamos un camino que esperamos que tenga continuidad.

Así las cosas, como consecuencia de esta investigación y de otras de carácter similar llevadas a cabo por el equipo de investigación Gentt (García Izquierdo, 2009 y 20II; García Izquierdo y Conde, 20r2; Muñoz Miquel, en proceso) se constata la necesidad de profundizar en el diseño de recursos documentales específicos para los traductores médicos en el contexto español. Por ello, a partir de los datos contenidos en el corpus Gentt 3.o, se planificó el diseño de una nueva plataforma web, que estará disponible en breve ${ }^{13}$, y que ofrecerá a los traductores diversos recursos de carácter lingüístico y documental. En concreto, como se avanza en Borja (20I2: I74), los contenidos elaborados hasta la fecha para el ámbito médico se refieren

r3 El diseño de esta plataforma es uno de los resultados del proyecto de investigación financiado por el Ministerio de Ciencia y Tecnología al equipo de investigación Gentt en el trienio 2009-20I2. específicamente a los géneros médico-jurídicos y en la plataforma se incluye tanto el catálogo de documentos más representativos en inglés y en español, como las fichas explicativas de las 161 características fundamentales de cada uno de ellos (denominación, aspectos formales, detalles de la situación comunicativa y macroestructura), un apartado de Normativa específica para la interpretación del ámbito, un apartado con una amplia selección de recursos documentales (en formato papel y en línea) y un apartado de Glosario de términos de elaboración propia. Todo ello constituye la base de datos para el buscador que incorpora la herramienta, que permite realizar búsquedas avanzadas en función de diferentes criterios.

El objetivo de la plataforma, y de las réplicas con géneros de otros ámbitos importantes en la traducción médica, como el de Información para pacientes ${ }^{\mathrm{I}}$, que ocupará nuestras próximas investigaciones, será proporcionar, pues, información adecuada a las expectativas de los usuarios, de acuerdo con las conclusiones de estudios como el que aquí hemos presentado.

RECIBIDO EN MARZO DE 2013

ACEPTADO EN JUNIO DE 2013

VERSIÓN FINAL DE MARZO DE 2013

\section{REFERENCIAS BIBLIOGRÁFICAS}

Borja, A. (2012). «Aproximación traductológica a los textos médico-jurídicos».Pan@cea, I3/36, pp. I67-175.

Ericsson, K.A. (1996). The road to excellence: The acquisition of expert performance in the arts and sciences, sports and games, Nueva Jersey: Lawrence Erlbaum Associated, Inc.

I4 El equipo acaba de obtener financiación del Ministerio de Economía y Competitividad para el trienio 20I3-20I6 (FFI2OI2-34200) para investigar en el ámbito de la información para pacientes. 
García-Izquierdo, I. (2009). Divulgación médica y traducción: El género Información para pacientes, Berna: Peter Lang.

García-Izquierdo, I. (20II). «La investigación cualitativa en traducción especializada: Una mirada a los ámbitos socioprofesionales». En: S. Cruces, A. Luna, M. Del Pozo y A. Álvarez, eds. 20Ir. Traducir en la frontera, Granada: Atrio, pp. 603-6ig.

García-Izquierdo, I. y Conde, T. (2012). «Investigating specialized translators: Corpus and Documentary sources». Ibérica, 23, pp. I3I-I57.

Lee-Jahnke, H. (1998). «Training in Medical Translation with Emphasis on German». En: H. Fischbach, ed. I998. Translation and Medicine, Ámsterdam: John Benjamins, pp. 8I-9I.

Muñoz, R. (20II). «Nomen mibi Legio est: A cognitive approach to Natural Translation». En: $\mathrm{M}^{\mathrm{a}}$ J. Blasco y A. Jiménez, eds. Interpreting... naturally, Fráncfort: Peter Lang, pp.35-66.

Muñoz Miquel, A. (20I2). «La traducció mèdica en la formació de postgrau a Espanya». I Congreso
Internacional de Investigación en Didáctica de la Traducción (DidTrad). 2I-22 de junio de 2012. Barcelona: Universitat Autònoma de Barcelona.

Muñoz Miquel, A. (en proceso). «La enseñanza de la traducción médica en España. Competencias y perfiles profesionales». Tesis doctoral. Universitat Jaume I, Castellón.

Muñoz, A., Montalt, V. y García-Izquierdo, I. (20II). «La investigación socioprofesional y la competencia traductora aplicadas a la pedagogía de la traducción médica». En: S. Cruces, A. Luna, M. Del Pozo y A. Álvarez, eds. (20II). Traducir en la frontera, Granada: Atrio, pp. Io3-I2I.

Montalt, V. y Gonzales-Davies, M. (2007). Medical translation step by step. Learning by Drafting, Mánchester: St. Jerome.

Montalt, V. y Suttleworth, M. eds., (2012). Translation and Knowledge. Mediation in Medical and Health Settings. Lingüistica Antverpiensia, New Series-Themes in Translation Studies, II. 